\title{
Stents Auto-Expansíveis Versus Balão-Expansíveis para Tratamento de Lesões em Vasos de Fino Calibre: Estudo com Ultra-Som Intracoronário Tridimensional
}

\author{
Daniel Chamié ${ }^{1}$, José Ribamar Costa Jr. ${ }^{1}$, Alexandre Abizaid ${ }^{1}$, Fausto Feres ${ }^{1}$, Fernando Devito ${ }^{2}$, \\ Luiz Alberto Mattos ${ }^{1}$, Rodolfo Staico', Andréa Abizaid', Luiz Fernando L. Tanajura', \\ Peter J. Fitzgerald ${ }^{3}$, Robert Whitbourn ${ }^{4}$, Amanda G. M. R. Sousa ${ }^{1}$, J. Eduardo Sousa ${ }^{1}$
}

\section{RESUMO}

Introdução: Intervenção coronária percutânea em vasos de fino calibre (VF) está associada a piores resultados imediatos e tardios, com elevadas taxas de reestenose. Estudos prévios têm sugerido que stents auto-expansíveis causam menos injúria vascular no momento do implante, com expansão de seus volumes com o tempo, gerando maiores áreas luminais que stents balão-expansíveis. A influência desses fenômenos em VF ainda é desconhecida. Objetivos: Avaliar as propriedades mecânicas e a eficácia do novo stent CardioMind ${ }^{\circledR}$ no tratamento de lesões em VF em comparação ao stent balão-expansível Multi-Link Pixel ${ }^{\circledast}$ (Pixel). Método: Treze pacientes portadores de lesões únicas primárias, $<14 \mathrm{~mm}$ de extensão, em artérias coronárias nativas $<2,5 \mathrm{~mm}$ de diâmetro, foram tratados com o stent CardioMind ${ }^{\circledR}$ e comparados a uma coorte histórica de 25 pacientes, com os mesmos critérios de inclusão, tratados com o stent Pixel ${ }^{\circledR}$. Ultrasom intracoronário (USIC) seriado foi realizado pós-procedimento e aos 7,3 $\pm 1,0$ meses. Resultados: A média das idades foi de $58,1 \pm 9,9$ anos, com $60,5 \%$ do sexo masculino e $39,4 \%$ diabéticos. Ambos os stents produziram volumes de hiperplasia neo-intimal (HNI) semelhantes (volume de $\mathrm{HNI}$ indexado: $1,45 \pm 0,46 \mathrm{~mm}^{3} / \mathrm{mm}$ para o CardioMind ${ }^{\circledR}$ vs. $1,66 \pm 1,02 \mathrm{~mm}^{3} / \mathrm{mm}$ para o Pixel $^{\oplus ;}$ $p=0,48)$. Entretanto, o stent CardioMind ${ }^{\circledR}$ apresentou expansão de $12 \%$ em seus volumes, gerando menor porcentual de obstrução volumétrica intra-stent $(31,94 \pm$ $8,19 \%$ vs. $39,90 \pm 4,72 \% ; p=0,0005)$. Nenhum dos tipos de stent causou injúria nos segmentos de borda. Conclu-

\section{SUMMARY}

Self-Expanding Versus Balloon-Expandable Stents for the Treatment of Small Coronary Vessels: a Three-dimensional Intracoronary Ultrasound Study

Background: Percutaneous coronary intervention in small vessels (SV) is associated with poor short- and long-term outcomes, with high rates of restenosis. Previous studies have suggested that self-expanding stents can cause less vessel injury at implantation, expanding their volumes over time, and leading to larger luminal areas than those of balloon-expandable stents. The influence of these phenomena on SV remains unknown. Objectives: To assess the mechanical properties and efficacy of the novel CardioMind ${ }^{\mathrm{TM}}$ stent in comparison with the balloon-expandable Multi-Link PixelTM (Pixel) stent in the treatment of SV. Methods: Thirteen patients with single, de novo, $<14 \mathrm{~mm}$ length lesions in native coronary arteries $<2.5 \mathrm{~mm}$ in diameter were treated with the CardioMind ${ }^{\mathrm{TM}}$ stent and compared with a historical cohort of 25 patients, with the same inclusion criteria, treated with the Pixel ${ }^{\mathrm{TM}}$ stent. Intravascular ultrasound (IVUS) was performed serially after the procedure and at $7.3 \pm 1.0$ months of follow-up. Results: Mean age was $58.1 \pm 9.9$ years; $60.5 \%$ were male and $39.4 \%$ were diabetic. Both stents produced similar neointimal hyperplasia $(\mathrm{NIH})$ volumes (indexed $\mathrm{NIH}$ volume: $1.45 \pm$ $0.46 \mathrm{~mm}^{3} / \mathrm{mm}$ for CardioMind ${ }^{\mathrm{TM}}$ versus $1.66 \pm 1.02 \mathrm{~mm}^{3} / \mathrm{mm}$ for PixelTM; $p=0.48$ ). However, the CardioMind ${ }^{\mathrm{TM}}$ stent presented a $12 \%$ expansion of its volume, leading to a

\footnotetext{
1 Instituto Dante Pazzanese de Cardiologia - São Paulo, SP.

2 Hospital São Domingos - Faculdade de Medicina da FIPA Catanduva, SP.

3 Stanford University Medical Center - Stanford, Califórnia, Estados Unidos.

${ }^{4}$ The Cardiovascular Research Center - St. Vincent's Hospital Melbourne, Austrália.

Correspondência: Alexandre Abizaid. Instituto Dante Pazzanese de Cardiologia - Av. Dr. Dante Pazzanese, 500 - São Paulo, SP

CEP 04038-003 - E-mail: aabizaid@uol.com.br

Recebido em: 13/6/2008 • Aceito em: 5/9/2008
} 
sões: A análise seriada com USIC demonstrou que os stents CardioMind ${ }^{\circledR}$ e Pixel $^{\circledR}$ produziram volumes semelhantes de tecido neo-intimal. Entretanto, a expansão continuada dos volumes do CardioMind ${ }^{\circledR}$ promoveu maior volume luminal e menor porcentual de obstrução intra-stent. Ambos os stents foram inócuos aos segmentos das bordas.

DESCRITORES: Contenedores. Angioplastia coronária transluminal percutânea. Ultra-sonografia. lesser in-stent volumetric obstruction $(31.94 \pm 8.19 \%$ versus $39.90 \pm 4.72 \% ; p=0.0005)$. Injury at the edges of the CardioMind $^{\mathrm{TM}}$ and Multi-Link Pixel ${ }^{\mathrm{TM}}$ stents were not detected. Conclusions: Serial volumetric IVUS showed that CardioMind ${ }^{\mathrm{TM}}$ and Pixe $^{\mathrm{TM}}$ stents produced similar volumes of neointimal tissue. However, continued expansion of CardioMind $^{\mathrm{TM}}$ stent generated larger luminal volumes and lower percent in-stent volumetric obstruction. The two stents were harmless to the edge segments.

DESCRIPTORS: Stents. Angioplasty, transluminal, percutaneous coronary. Ultrasonography.

tados com o stent balão-expansível Multi-Link Pixel ${ }^{\circledR}$, ambos desenvolvidos para o tratamento de lesões em artérias coronárias de fino calibre.

\section{Seleção de pacientes} de reestenose mesmo com o uso de stents farmacológicos ${ }^{4,5}$.

Contudo, dependendo da definição empregada, a intervenção em vasos de fino calibre é cenário freqüente, podendo corresponder a $35 \%$ a $67 \%$ de todos os procedimentos de $\mathrm{ICP}^{6}$.

Nesse sentido, novas tecnologias dedicadas especificamente para esse subgrupo de alta complexidade têm sido criadas.

O sistema de stent coronário CardioMind ${ }^{\circledR}$, que consiste de um stent auto-expansível de nitinol montado sobre uma corda-guia 0,014" e com muito baixo perfil, foi recentemente desenvolvido para a obtenção de acesso a artérias coronárias apresentando anatomia complexa e para o tratamento de lesões em vasos de fino calibre ${ }^{7}$.

O stent CardioMind ${ }^{\circledR}$ foi testado pela primeira vez em seres humanos numa coorte pequena de pacientes, demonstrando resultados bastante promissores na redução da formação da hiperplasia neo-intimal $(\mathrm{HNI})^{8}$, mecanismo preponderante de reestenose com o uso dos stents coronários.

Entretanto, não se sabe se esse dispositivo pode fornecer vantagens em comparação aos modelos de stents disponíveis comercialmente. Neste estudo, portanto, tivemos como objetivo apresentar uma comparação, com o uso do ultra-som intracoronário (USIC), entre a aplicação clínica do stent CardioMind ${ }^{\circledR}$ e do stent Multi-Link Pixel ${ }^{\circledR}$, balão-expansível e também de baixo perfil, no tratamento das lesões em vasos de fino calibre.

\section{MÉTODO}

\section{Desenho do estudo}

Este é um estudo não-randomizado que compara, por meio do USIC, a eficácia do stent auto-expansível CardioMind ${ }^{\circledR}$ a uma coorte histórica de pacientes tra-
Durante o período de maio de 2006 a janeiro de 2007, 21 pacientes foram tratados com o stent CardioMind ${ }^{\circledR}$. Os critérios de inclusão foram:

- portadores de angina estável (classificação da Canadian Cardiovascular Society I-IV), angina instável (classificação de Braunwald IB, IC, IIB, IIC) ou isquemia silenciosa;

- presença de lesão única, de novo, com extensão menor que $14 \mathrm{~mm}$ e diâmetro de estenose $\geq 50 \%$ e $<100 \%$, em artérias coronárias nativas com diâmetro de referência entre $2,0 \mathrm{~mm}$ e $2,5 \mathrm{~mm}$ por estimativa visual;

- fluxo coronário TIMI $\geq \mathrm{II}$.

O grupo controle, com os mesmos critérios de inclusão e pareados com relação às características clínicas e angiográficas de base, foi constituído de 30 pacientes tratados com o stent Multi-Link Pixel ${ }^{\circledR}$ no período de abril a dezembro de 2003.

Os resultados das análises angiográfica e ultrasonográfica desses dois dispositivos foram apresentados previamente ${ }^{9}$.

A presente análise inclui os pacientes que foram submetidos a realização de USIC seriado imediatamente após o procedimento e no seguimento a médio prazo.

O protocolo do estudo foi aprovado pelo Comitê de Ética em Pesquisa de nossa instituição e todos os pacientes incluídos assinaram termo de consentimento informado.

\section{Características dos dispositivos estudados}

Sistema de Stent CardioMind ${ }^{\circledR}$ : O sistema de stent coronário CardioMind ${ }^{\circledR}$ (CardioMind Inc., Califórnia, Estados Unidos) já foi previamente descrito em deta$\mathrm{Ihes}^{7,8}$. Resumidamente, consiste de um stent auto-ex- 

com Ultra-Som Intracoronário Tridimensional. Rev Bras Cardiol Invas. 2008;16(3):341-352.

pansível de nitinol, de células fechadas em formato de diamante, com hastes extremamente finas $(0,0024 ")$, incorporado a uma corda-guia 0,014", que serve de plataforma para a "entrega" do stent, conferindo perfil extremamente baixo e alta flexibilidade. Esse conjunto é acoplado a um sistema de liberação que mantém o stent mecanicamente restrito a seu menor diâmetro por meio de um mecanismo de contenção retrátil, evitando sua expansão espontânea. Uma corrente de energia elétrica menor que $0,5 \mathrm{~mA}$, proveniente de uma fonte de bateria bipolar, promove a eletrólise do mecanismo de contenção e, conseqüentemente, a liberação do stent.

Stent Multi-Link Pixel ${ }^{\circledR}$ : O stent Multi-Link Pixel ${ }^{\circledR}$ (Guidant Corp., Califórnia, Estados Unidos) é um stent balão-expansível, feito de aço inoxidável em anéis ondulados de cinco pontas, caracterizado pela baixa espessura de suas hastes $(0,0039 ")$. O stent vem "prémontado" em um balão de baixa complacência, com tecnologia conhecida como S.T.E.P. (Short Transitional Edge Protection), minimizando a área de injúria ao vaso fora do segmento do stent.

A Figura 1 ilustra as principais características dos dois sistemas de stent coronário.

\section{Objetivos}

O desfecho primário de eficácia deste estudo é a comparação do porcentual de obstrução volumétrica do stent pela $\mathrm{HNI}$ entre os dois dispositivos. Como desfechos secundários, procurou-se investigar as propriedades mecânicas de funcionamento das duas endopróteses, bem como sua influência no remodelamento vascular e nos segmentos das bordas.

\section{Procedimento de intervenção coronária}

Todos os pacientes foram pré-tratados com aspirina e um tienopiridínico. Aspirina era administrada na dose de $200 \mathrm{mg}, 24$ horas antes do procedimento, e mantida indefinidamente. O pré-tratamento com clopidogrel foi feito com 300 mg 24 horas antes do procedimento. Caso o tienopiridínico utilizado fosse a ticlopidina, o pré-tratamento era feito na dose de $500 \mathrm{mg}$ por dia pelo menos 48 horas antes do procedimento. A terapia de antiagregação plaquetária dupla foi mantida por três meses para pacientes submetidos a implante de stent CardioMind ${ }^{\circledR} \mathrm{e}$ por um mês para aqueles submetidos a implante do stent Multi-Link Pixel ${ }^{\circledR}$.

Durante o procedimento, todos os pacientes receberam heparina não-fracionada na dose de 2.500 UI na via do introdutor arterial. No momento da passagem da corda-guia, administrava-se mais uma dose intravenosa, para complementar a dose total ajustada pelo peso do paciente, de $100 \mathrm{UI} / \mathrm{kg}$. Caso necessário, bolos adicionais eram administrados após a primeira
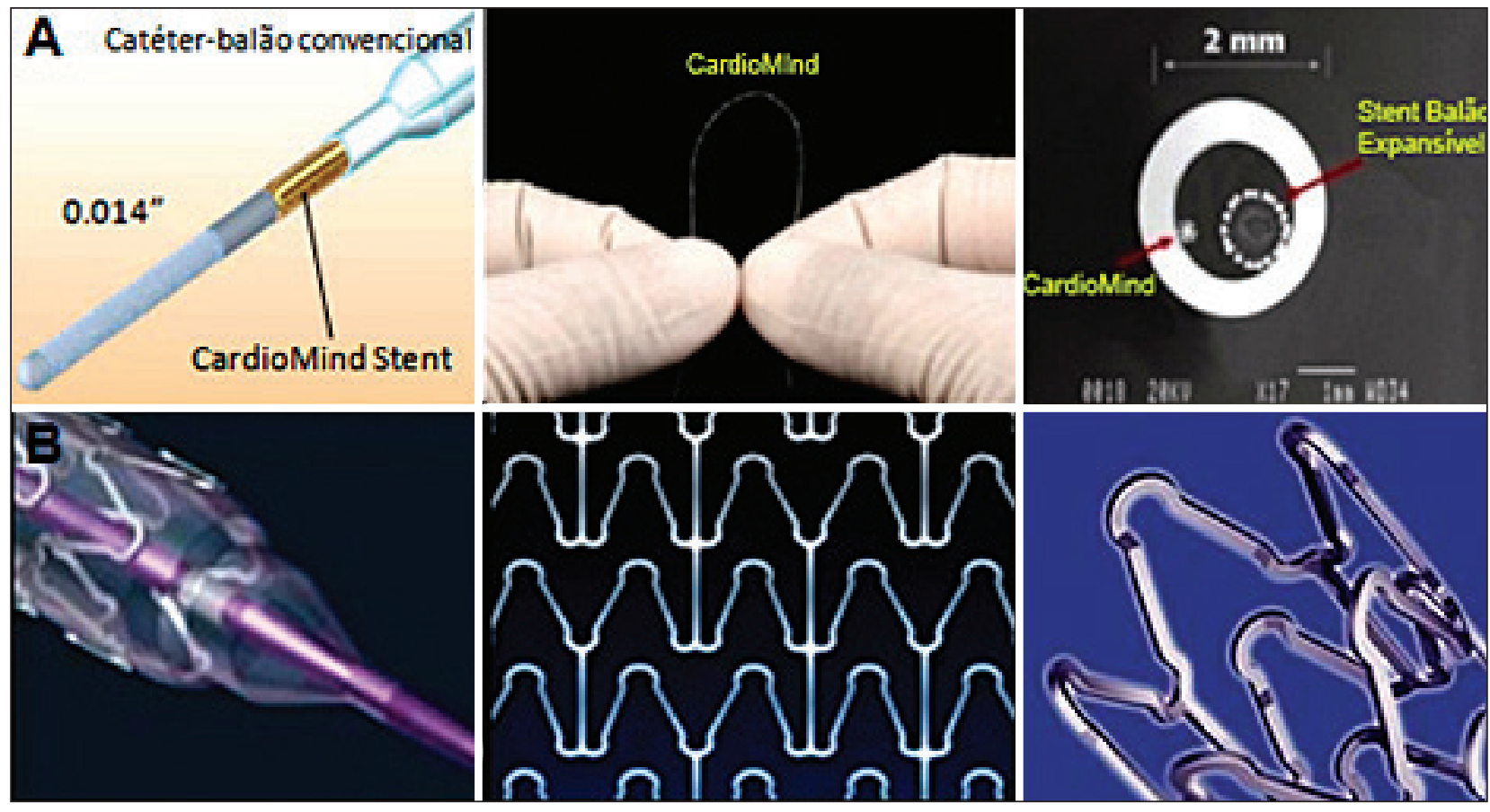

Figura 1 - Principais características dos dois sistemas de stent coronário estudados. Em A, o stent CardioMind". À esquerda, stent "montado" sobre o guia e a presença do cateter-balão de angioplastia coronária sobre o conjunto "guia + stent". O segmento do guia que fica sob o stent tem perfil 0,006", permitindo que todo o conjunto tenha perfil 0,014". Na figura central, sua excelente flexibilidade. Na figura da direita, observase o baixo perfil do conjunto dentro de um cateter-guia 6 French, comparado com um stent balão-expansível convencional. Em B, o stent MultiLink Pixel ${ }^{\circledR}$. Na figura da esquerda, stent "montado" sobre o cateter-balão com a tecnologia S.T.E.P. (Short Transitional Edge Protection). Na figura central, seu desenho em anéis ondulados de cinco pontas. Na figura da direita, a configuração do stent totalmente expandido. 
hora do procedimento, para manutenção do tempo de coagulação ativado entre 250 e 350 segundos. O uso de inibidores de glicoproteína Ilb/Illa não era obrigatório e ficou a critério do operador.

A angiografia inicial era realizada em pelo menos duas projeções ortogonais, após administração de $100 \mu \mathrm{g}$ a $200 \mu \mathrm{g}$ de nitroglicerina intracoronária.

A pré-dilatação da lesão-alvo era obrigatória, utilizando-se cateteres-balão com diâmetro pelo menos 0,5 $\mathrm{mm}$ menores que o diâmetro de referência do vaso e extensão menor que o tamanho do stent escolhido.

No caso do stent CardioMind ${ }^{\circledR}$, a corda-guia 0,014" é a própria plataforma de carreamento do stent, que fica posicionada proximal aos $2 \mathrm{~cm}$ a $3 \mathrm{~cm}$ radiopacos da porção distal do guia. Uma segunda marca radiopaca delimita a borda proximal do stent. Utiliza-se, então, essas duas marcações radiopacas para posicionamento do stent, encobrindo a lesão-alvo antes da realização da pré-dilatação. O stent "montado" sobre a cordaguia não aumenta seu perfil, que se mantém de 0,014", sendo totalmente compatível com os cateteres-balão utilizados na rotina. Após o posicionamento do stent, o cateter-balão é avançado sobre o conjunto guiastent e posicionado no ponto da lesão. Após insuflação do balão e dilatação da lesão-alvo, recua-se o balão para a parte proximal ao segmento a ser tratado, aciona-se o sistema de liberação do stent, promovendo a expansão do mesmo, e, caso seja necessária pós-dilatação para otimização do implante, pode-se avançar o mesmo cateter-balão da pré-dilatação por sobre o guia (desta vez por dentro do stent) e realizar novas insuflações, minimizando sobremaneira a troca de materiais e a manipulação do vaso coronário.

O implante do stent Multi-Link Pixe ${ }^{\circledR}$ foi realizado de acordo com as técnicas padrão de implante de stent coronário balão-expansível.

Os stents CardioMind ${ }^{\circledR}$ estavam disponíveis nos tamanhos 2/19 mm e 2,5/20 mm. Stents de 2/12 mm, 2,5/12 mm e 2,5/13 mm estavam disponíveis apenas caso fosse necessário implante de novo stent por dissecção de bordas e/ou cobertura incompleta da lesão. Os stents Multi-Link Pixel ${ }^{\circledR}$ estavam disponíveis nos diâmetros de $2 \mathrm{~mm}, 2,25 \mathrm{~mm}$ e 2,5 mm, com extensão de $8 \mathrm{~mm}, 13 \mathrm{~mm}, 18 \mathrm{~mm}, 23 \mathrm{~mm}$ e $28 \mathrm{~mm}$.

O uso de apenas um stent por lesão era recomendado. Caso houvesse necessidade de implante de novo stent, este deveria ser disposto com sobreposição de $2 \mathrm{~mm}$ a $3 \mathrm{~mm}$ ao primeiro stent. Em caso de expansão inadequada, com lesão residual $>20 \%$, o stent poderia ser pós-dilatado com cateter-balão mais curto que o stent implantado.

\section{Análises quantitativa e qualitativa do ultra-som intracoronário}

As imagens do USIC pós-procedimento e no seguimento foram adquiridas utilizando-se transdutor de elemento único, rotacional, com freqüência de $40 \mathrm{MHz}$, envolto numa bainha com perfil 2,6 French, com recuo motorizado em sistema de tração automática à velocidade de $0,5 \mathrm{~mm} / \mathrm{seg}$ undo e conectado a um scanner comercial (Clear View ou Galaxy 2, Boston Scientific Corp., Nattick, Massachusetts, Estados Unidos).

As imagens foram digitalizadas para análise quantitativa off-line, de acordo com os critérios do American College of Cardiology Clinical Expert Consensus Document on IVUS ${ }^{10}$.

Para realização da análise volumétrica, foi feita a reconstrução tridimensional das imagens do USIC com a utilização de um programa de planimetria computadorizada comercialmente disponível (EchoPlaque 3,0, INDEC Systems Inc. - Mountain View, Califórnia, Estados Unidos).

No segmento tratado por stent, as áreas do lúmen, do stent e do vaso (membrana elástica externa) foram determinadas, a cada milímetro, por planimetria computadorizada. A área da HNI foi calculada como a área do stent menos a área do lúmen na avaliação tardia. Utilizando-se a regra de Simpson, foram calculados os volumes do lúmen, do stent e do vaso. O volume de $\mathrm{HNI}$ foi determinado pela diferença entre os volumes do stent e do lúmen no seguimento. Como a extensão do segmento coberto por stent não era idêntica entre os pacientes dos dois grupos, os dados volumétricos da HNI foram corrigidos de acordo com a extensão do segmento coberto por stent para cada paciente (volume de $\mathrm{HNI}$ indexado). Os volumes indexados eram calculados como: volume absoluto da HNI/comprimento do stent medido pelo USIC ${ }^{11}$. O porcentual de obstrução volumétrica intra-stent é calculado como a razão entre o volume da HNI e o volume do stent $x$ 100. A diferença entre os volumes do vaso e do stent foi definida como o volume do espaço peri-stent.

$\mathrm{Na}$ análise qualitativa do segmento intra-stent, aposição incompleta do stent foi definida como a separação de pelo menos uma haste do stent da parede vascular, com presença de fluxo de sangue por trás das hastes, sem envolver uma área de bifurcação com outro ramo $^{12}$. Aposição incompleta do stent foi, ainda, classificada como persistente (presente na análise pósprocedimento e na análise tardia), tardia ou adquirida (ausente na análise pós-procedimento e presente na avaliação tardia), e resolvida (presente na análise pósprocedimento e ausente na avaliação tardia) ${ }^{13}$.

Para determinação do chamado "efeito de bordas", análise quantitativa foi ainda realizada nos segmentos localizados $5 \mathrm{~mm}$ proximais e distais ao stent, configurando as bordas proximal e distal, respectivamente. Nos casos de presença de calcificação importante com formação de sombra acústica, presença de ramos nos segmentos das bordas ou corridas do USIC menores que os $5 \mathrm{~mm}$ pré-definidos para cada borda, esses segmentos foram excluídos da análise. Para cada borda, os volumes da membrana elástica externa, lúmen e placa foram determinados também pela regra de 

com Ultra-Som Intracoronário Tridimensional. Rev Bras Cardiol Invas. 2008;16(3):341-352.

Simpson. A carga de placa foi calculada como a razão entre o volume da placa/volume do vaso x 100 .

A variação $(\Delta)$ dos volumes foi calculada como o volume obtido no seguimento tardio menos o volume pós-procedimento.

\section{Análise estatística}

As variáveis categóricas foram expressas como freqüências absolutas e porcentuais. As variáveis contínuas foram apresentadas como média e desvio padrão. Para comparação das variáveis categóricas, foram utilizados os testes de qui-quadrado e exato de Fisher. As variáveis contínuas foram comparadas por meio do teste $t$ de Student, pareado ou não-pareado. $O$ valor de $p$ bicaudal foi considerado significativo quando $<0,05$.

A análise estatística foi realizada com o uso do programa Statistical Package for Social Sciences (SPSS, Inc.) versão 13.0 para Windows.

\section{RESULTADOS}

No período de maio de 2006 a janeiro de 2007, 21 pacientes foram submetidos a implante do stent CardioMind $^{\circledR}$. Avaliação imediata pós-implante do stent com USIC foi realizada em 18 desses pacientes. Na avaliação tardia, ao final de um seguimento médio de 7,3 \pm 1,0 meses, 13 pacientes tinham avaliações seriadas (pós-implante e seguimento) com USIC adequadas para análise.

No grupo controle, no período de abril a dezembro de 2003, 30 pacientes foram submetidos a implante do stent Multi-Link Pixel ${ }^{\circledR}$, com avaliação imediata pós-implante do stent por USIC realizada em 27 pacientes (3 tinham imagens inadequadas para análise). Após o mesmo período de seguimento, 25 pacientes tinham avaliações do USIC seriadas adequadas para análise.

A média das idades da população total $(n=38)$ foi de 58,1 \pm 9,9 anos, sendo $23(60,5 \%)$ pacientes do sexo masculino. Trinta $(78,9 \%)$ pacientes eram portadores de hipertensão arterial sistêmica e 15 (39,4\%), de diabetes melito. As características clínicas de base foram semeIhantes entre os grupos e são apresentadas na Tabela 1.

Os vasos mais freqüentemente tratados foram a artéria coronária direita e seus ramos distais $(46,1 \%)$, no grupo CardioMind ${ }^{\circledR}$, e a artéria descendente anterior em seus segmentos médio e distal (48\%), no grupo Multi-Link Pixel ${ }^{\circledR}$. As lesões tratadas eram predominantemente dos tipos B2 e C (segundo classificação do American College of Cardiology/American Heart Association $[\mathrm{ACC} / \mathrm{AHA}]^{14}$ ), em ambos os grupos de tratamento. Os pacientes tratados com o stent CardioMind ${ }^{\circledR}$ possuíam vasos com diâmetro de referência significativamente menor $(2,23 \pm 0,19 \mathrm{~mm}$ vs. 2,42 \pm $0,17 \mathrm{~mm} ; \mathrm{p}=0,003)$. Por outro lado, a extensão das lesões foi maior no grupo dos pacientes tratados com o stent Multi-Link Pixel ${ }^{\circledast}(13,36 \pm 2,60 \mathrm{~mm}$ vs. 10,46 \pm
3,80 mm; $p=0,005)$. Entretanto, a extensão total do segmento coberto por stent foi semelhante entre os dois grupos $\left(16,44 \pm 5,12 \mathrm{~mm}\right.$ para o CardioMind ${ }^{\circledR}$ vs. 17,40 $\pm 4,30 \mathrm{~mm}$ para o Multi-Link Pixel $\left.{ }^{\circledR} ; \mathrm{p}=0,54\right)$. A Tabela 2 apresenta as características angiográficas de base e os dados relacionados ao procedimento para ambos os grupos.

\section{Análise intra-stent pelo ultra-som intracoronário}

A Tabela 3 apresenta os dados da análise volumétrica do USIC nos períodos pós-procedimento e no seguimento para os dois grupos de tratamento. Os parâmetros avaliados na análise ultra-sonográfica pós-procedimento são semelhantes entre os dois grupos. No seguimento médio de sete meses, os stents CardioMind $^{\circledR}$ e Multi-Link Pixel ${ }^{\circledR}$ apresentaram volumes de HNI semelhantes (volume de HNI indexado: $1,45 \pm 0,46 \mathrm{~mm}^{3} / \mathrm{mm}$ para o CardioMind ${ }^{\circledR}$ vs. $1,66 \pm$ $1,02 \mathrm{~mm}^{3} / \mathrm{mm}$ para o Multi-Link Pixel $\left.{ }^{\circledR} ; \mathrm{p}=0,48\right)$. Entretanto, esse volume similar de HNI gerou um porcentual de obstrução volumétrica intra-stent significativamente menor no stent CardioMind ${ }^{\circledR}(31,94 \pm 8,19 \%$ vs. $39,90 \pm 4,72 \% ; p=0,0005)$.

Nas Figuras 2 e 3, observa-se que houve expansão significativa de $12 \%$ nos volumes do stent CardioMind $^{\circledR}$ ao final de sete meses em comparação com o stent Multi-Link Pixel ${ }^{\circledR}$ (variação de 9,18 \pm 9,77 $\mathrm{mm}^{3}$ vs. $\left.1,23 \pm 12,59 \mathrm{~mm}^{3} ; \mathrm{p}=0,04\right)$. Percebe-se, ainda, que esse fenômeno ocorreu por ocupação do espaço peri-stent e não por remodelamento vascular positivo. Houve maior redução do espaço peri-stent no grupo CardioMind $^{\circledR}\left(8,92 \pm 9,68 \mathrm{~mm}^{3}\right.$ vs. $1,94 \pm 16,73 \mathrm{~mm}^{3}$; $\mathrm{p}=0,17)$, e praticamente nenhuma variação nos volumes do vaso $\left(0,26 \pm 15,80 \mathrm{~mm}^{3}\right.$ para o CardioMind ${ }^{\circledR}$ vs. $-0,70 \pm 16,34 \mathrm{~mm}^{3}$ para o Multi-Link Pixel $\left.{ }^{\circledR ;} p=0,86\right)$. A expansão contínua do stent CardioMind $^{\circledR}$ ao longo do seguimento médio de sete meses foi capaz de acomodar melhor a mesma quantidade de HNI formada pelos dois stents, promovendo menor porcentual de obstrução volumétrica do stent CardioMind ${ }^{\circledR} \mathrm{e}$, conseqüentemente, menores reduções em seus volumes luminais (redução média do lúmen de 18,38 \pm $12,71 \mathrm{~mm}^{3}$ vs. $\left.25,83 \pm 20,96 \mathrm{~mm}^{3} ; \mathrm{p}=0,24\right)$.

Ademais, na análise qualitativa pelo USIC, observou-se incidência de 6 (46\%) casos de má aposição das hastes do stent CardioMind ${ }^{\circledR}$ à parede do vaso. Ao final do seguimento médio de sete meses, houve resolução da má aposição em 50\% dos casos. Não se detectou nenhum caso de má aposição adquirida. No grupo dos pacientes tratados com o stent Multi-Link Pixel $^{\circledR}$, não se detectou nenhum caso de má aposição das hastes do stent em qualquer dos períodos analisados.

\section{Análise do segmento de bordas pelo ultra-som intracoronário}

Do total de 38 pacientes (76 bordas) incluídos no estudo, $16(21 \%)$ bordas em 11 pacientes foram ex- 
TABELA 1

Características clínicas de base

\begin{tabular}{lccc}
\hline Variáveis & $\begin{array}{c}\text { CardioMind } \\
\mathbf{n = 1 3}\end{array}$ & $\begin{array}{c}\text { Multi-Link Pixel }^{\circledR} \\
\mathbf{n = 2 5}\end{array}$ & $\mathbf{p}$ \\
\hline Idade, anos & $62,60 \pm 12$ & $56,58 \pm 8,85$ & 0,08 \\
Sexo masculino & $8(61,5 \%)$ & $15(60 \%)$ & $>0,99$ \\
Hipertensão arterial & $10(76,9 \%)$ & $20(80 \%)$ & $>0,99$ \\
Diabetes melito & $6(46,1 \%)$ & $9(36 \%)$ & 0,72 \\
Dislipidemia & $12(92,3 \%)$ & $17(68 \%)$ & 0,12 \\
Tabagismo atual & $5(38,4 \%)$ & $8(32 \%)$ & 0,73 \\
IAM prévio & $5(38,4 \%)$ & $10(40 \%)$ & $>0,99$ \\
ICP prévia & $4(30,8 \%)$ & $9(36 \%)$ & $>0,99$ \\
CRVM prévia & $1(7,6 \%)$ & $1(4 \%)$ & $>0,99$ \\
\hline
\end{tabular}

CRVM = cirurgia de revascularização do miocárdio; IAM = infarto agudo do miocárdio; ICP = intervenção coronária percutânea; $\mathrm{n}=$ número de pacientes.

TABELA 2

Características angiográficas de base e dados do procedimento

\begin{tabular}{|c|c|c|c|}
\hline Variáveis & $\begin{array}{l}\text { CardioMind }^{\circledR} \\
\quad n=13\end{array}$ & $\begin{array}{c}\text { Multi-Link Pixel }^{\circledR} \\
n=25\end{array}$ & $\mathbf{p}$ \\
\hline \multicolumn{4}{|l|}{ Vaso-alvo } \\
\hline Descendente anterior & $2(15,3 \%)$ & $7(28 \%)$ & 0,45 \\
\hline Ramo diagonal & $1(7,8 \%)$ & $5(20 \%)$ & 0,64 \\
\hline Circunflexa & $1(7,8 \%)$ & $5(20 \%)$ & 0,64 \\
\hline Ramo obtuso marginal & $2(15,3 \%)$ & $4(16 \%)$ & $>0,99$ \\
\hline Artéria coronária direita & $3(23 \%)$ & $3(12 \%)$ & 0,39 \\
\hline Ramo ventricular posterior & 0 & $1(4 \%)$ & $>0,99$ \\
\hline Descendente posterior & $3(23 \%)$ & 0 & 0,03 \\
\hline Ramo intermédio & $1(7,8 \%)$ & 0 & 0,34 \\
\hline Lesões tipo B2/C** & $10(76,9 \%)$ & $16(64 \%)$ & 0,48 \\
\hline $\mathrm{DRV}, \mathrm{mm}$ & $2,23 \pm 0,19$ & $2,42 \pm 0,17$ & 0,003 \\
\hline $\mathrm{DLM}, \mathrm{mm}$ & $0,60 \pm 0,24$ & $0,72 \pm 0,26$ & 0,17 \\
\hline Diâmetro de estenose,\% & $72,69 \pm 11,99$ & $70,40 \pm 10,62$ & 0,54 \\
\hline Extensão da lesão, mm & $10,46 \pm 3,80$ & $13,36 \pm 2,60$ & 0,005 \\
\hline Diâmetro dos stents, mm & $2,43 \pm 0,17$ & $2,49 \pm 0,05$ & 0,10 \\
\hline Relação stent/artéria & $1,09 \pm 0,13$ & $1,03 \pm 0,07$ & 0,07 \\
\hline Extensão total do stent, mm & $16,44 \pm 5,12$ & $17,40 \pm 4,3$ & 0,54 \\
\hline Razão da extensão stent/lesão & $1,63 \pm 0,44$ & $1,36 \pm 0,48$ & 0,09 \\
\hline Pós-dilatação & $13(100 \%)$ & $4(16 \%)$ & $<0,0001$ \\
\hline Pressão de insuflação máxima, atm & $14,50 \pm 3,50$ & $14,58 \pm 1,90$ & 0,92 \\
\hline Relação diâmetro balão/artéria & $1,2 \pm 0,2$ & $1,1 \pm 0,2$ & 0,15 \\
\hline
\end{tabular}

cluídas da análise final, em função de um ou mais dos seguintes motivos: qualidade da imagem inadequada para análise $(7,8 \%)$, extensão incompleta - menor que $5 \mathrm{~mm}(15,7 \%)$, e presença de ramo significativo no segmento das bordas $(5,2 \%)$. Assim, a análise da bor- da proximal foi possível em $12(92,3 \%)$ pacientes no grupo CardioMind ${ }^{\circledR}$ e em 18 (72\%) no grupo MultiLink Pixel ${ }^{\circledR}$. A análise da borda distal foi possível em $10(76,9 \%)$ pacientes no grupo CardioMind ${ }^{\circledR}$ e em 20 $(80 \%)$ no grupo Multi-Link Pixel ${ }^{\circledR}$. 
TABELA 3

Resultados da análise volumétrica pelo ultra-som intracoronário do segmento intra-stent

\begin{tabular}{|c|c|c|c|}
\hline & $\begin{array}{l}\text { CardioMind }^{\oplus} \\
\quad n=13\end{array}$ & $\begin{array}{c}\text { Multi-Link Pixel }^{\circledR} \\
\quad n=25\end{array}$ & $\mathbf{p}^{*}$ \\
\hline \multicolumn{4}{|l|}{ Extensão analisada, mm } \\
\hline Pós-procedimento & $19,16 \pm 1,84$ & $17,20 \pm 4,72$ & 0,16 \\
\hline Seguimento & $19,07 \pm 1,12$ & $17,28 \pm 4,83$ & 0,19 \\
\hline $\mathrm{p}^{* *}$ & 0,88 & 0,95 & \\
\hline \multicolumn{4}{|l|}{ Volume do vaso $\left(\mathrm{mm}^{3}\right)$} \\
\hline Pós-procedimento & $159,88 \pm 34,30$ & $162,34 \pm 54,41$ & 0,88 \\
\hline Seguimento & $160,14 \pm 29,30$ & $161,64 \pm 53,54$ & 0,92 \\
\hline$p^{* *}$ & 0,98 & 0,96 & \\
\hline \multicolumn{4}{|l|}{ Volume do stent $\left(\mathrm{mm}^{3}\right)$} \\
\hline Pós-procedimento & $76,60 \pm 17,05$ & $72,32 \pm 26,61$ & 0,60 \\
\hline Seguimento & $85,78 \pm 15,48$ & $73,56 \pm 28,17$ & 0,15 \\
\hline $\mathrm{p}^{* *}$ & 0,16 & 0,87 & \\
\hline \multicolumn{4}{|l|}{ Volume do lúmen (mm³) } \\
\hline Pós-procedimento & $76,55 \pm 17,05$ & $72,32 \pm 26,61$ & 0,60 \\
\hline Seguimento & $58,17 \pm 11,45$ & $46,50 \pm 27,02$ & 0,14 \\
\hline$p^{* *}$ & 0,004 & 0,001 & \\
\hline \multicolumn{4}{|l|}{ Volume peri-stent $\left(\mathrm{mm}^{3}\right)$} \\
\hline Pós-procedimento & $83,28 \pm 19,62$ & $90,02 \pm 33,53$ & 0,51 \\
\hline Seguimento & $74,36 \pm 16,87$ & $88,08 \pm 29,11$ & 0,12 \\
\hline $\mathrm{p}^{* *}$ & 0,22 & 0,82 & \\
\hline Volume de $\mathrm{HNI}$ indexado (mm³/mm) & $1,45 \pm 0,46$ & $1,66 \pm 1,02$ & 0,48 \\
\hline Obstrução volumétrica intra-stent (\%) & $31,94 \pm 8,19$ & $39,90 \pm 4,72$ & 0,0005 \\
\hline
\end{tabular}

* Teste $t$ de Student não pareado. ** Teste $t$ de Student pareado.

$\mathrm{HNI}=$ hiperplasia neo-intimal; $\mathrm{n}=$ número de pacientes.

A Tabela 4 apresenta os resultados da análise ultra-sonográfica das bordas proximais e a Tabela 5, os resultados da análise das bordas distais.

Os pacientes tratados com o stent CardioMind ${ }^{\circledR}$ possuíam vasos com diâmetro de referência angiográfico significativamente menor $(2,23 \pm 0,19 \mathrm{~mm}$ vs. 2,42 \pm $0,17 \mathrm{~mm} ; \mathrm{p}=0,003)$. Portanto, na análise com USIC pós-procedimento, observou-se tendência a menor volume do vaso nas bordas proximais $\left(42,12 \pm 12,51 \mathrm{~mm}^{3}\right.$ vs. $\left.53,72 \pm 19,68 \mathrm{~mm}^{3} ; \mathrm{p}=0,06\right)$ e distais $(31,63 \pm$ $11,0 \mathrm{~mm}^{3}$ vs. $\left.39,90 \pm 11,47 \mathrm{~mm}^{3} ; p=0,06\right)$ quando comparados aos pacientes tratados com o stent MultiLink Pixel ${ }^{\circledR}$. Não se observou diferença estatística entre os volumes da placa e do lúmen, bem como nos diâmetros luminais mínimos e carga de placa, entre os dois grupos na análise pós-procedimento.

Apesar de o valor absoluto da área luminal mínima ser significativamente menor no grupo CardioMind ${ }^{\circledR}$, na análise tardia da borda distal $\left(2,49 \pm 0,79 \mathrm{~mm}^{2}\right.$ vs. $\left.3,50 \pm 1,07 \mathrm{~mm}^{2} ; p=0,01\right)$, não se observou variação significativa nos volumes do vaso, placa e lúmen entre os dois grupos de tratamento, no seguimento tardio, nas bordas proximal e distal (Figura 4).

Além disso, não foram detectadas diferenças significativas quando comparadas as variações nos volumes (vaso, placa e lúmen), na área luminal mínima e na carga de placa entre as bordas proximais e distais dos dois stents estudados (Tabela 6).

Na avaliação qualitativa pelo USIC não foram observadas dissecções de bordas em nenhum dos dois grupos.

\section{DISCUSSÃo}

Na presente análise, embora tanto o stent balãoexpansível como o auto-expansível tenham produzido similar quantidade de tecido neo-intimal em seu interior, o remodelamento positivo do stent no período entre o implante e o novo reestudo foi responsável por melhor acomodação desse tecido neoformado, resultando em maior volume luminal ao final de sete meses de evolução. No que se refere à injúria de bordas, ambos os stents avaliados mostraram-se inócuos nesse segmento. 


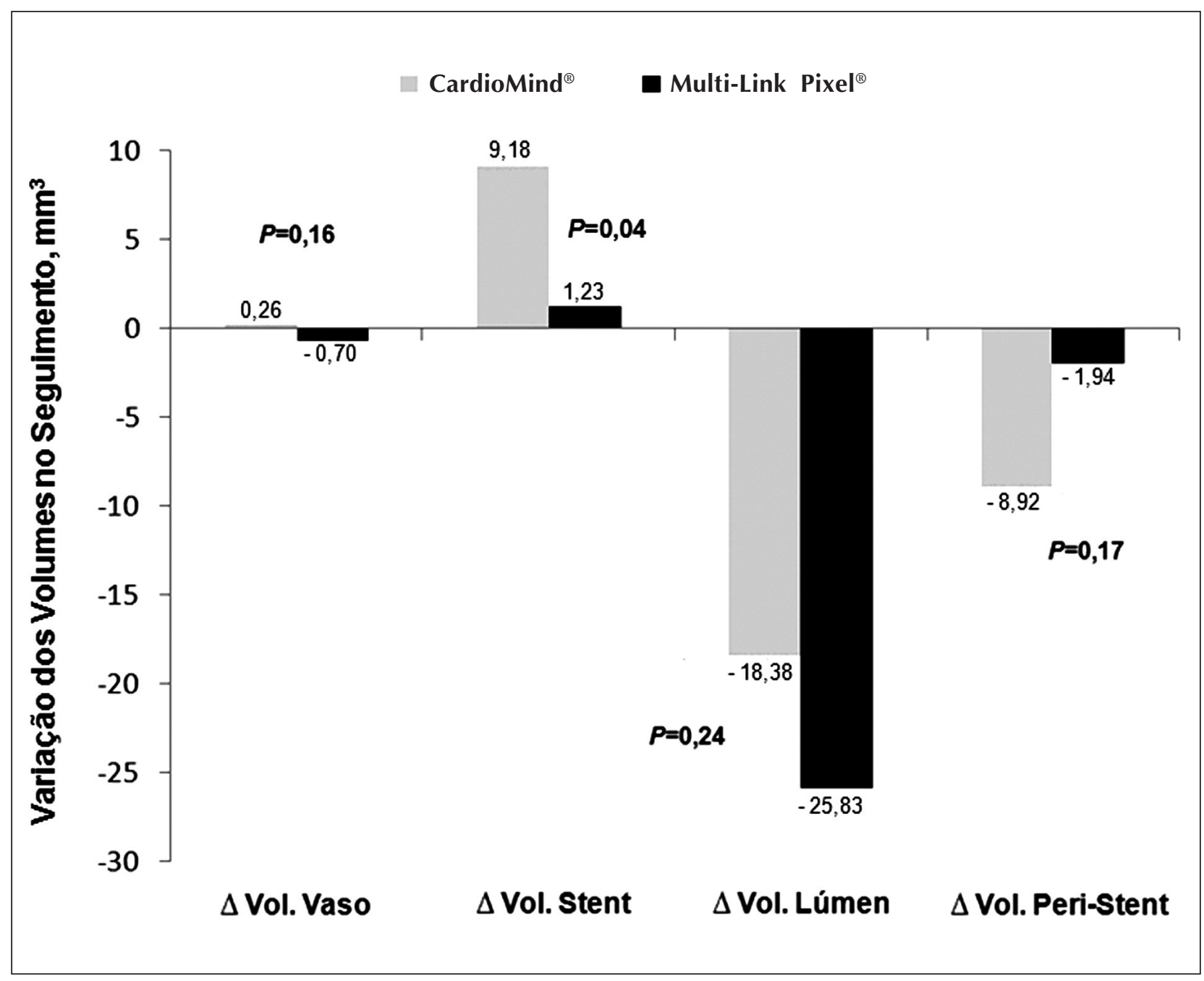

Figura 2 - Representação gráfica das variações volumétricas no segmento intra-stent na avaliação tardia com o USIC.

$\Delta$ Vol. = variação do volume.

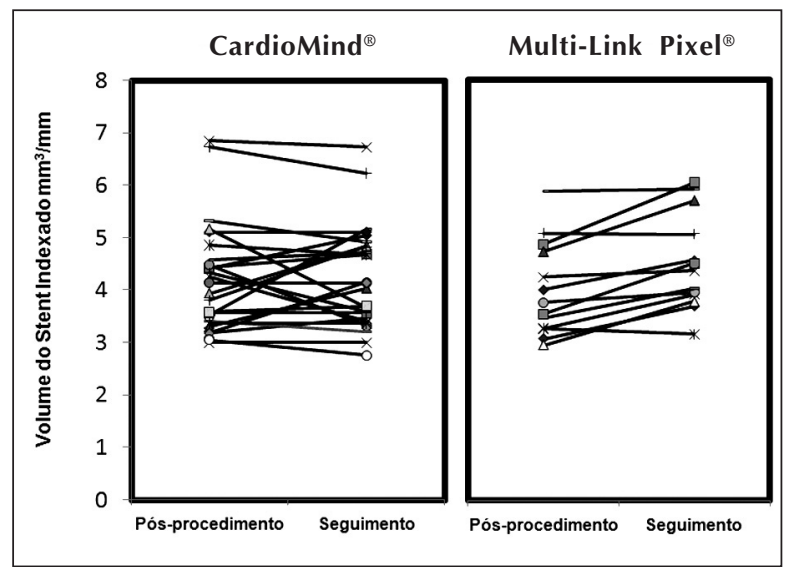

Figura 3 - Variações dos volumes dos stents Multi-Link Pixel ${ }^{\circledR} \mathrm{e}$ CardioMind ${ }^{\circledR}$ entre os períodos pós-procedimento e seguimento tardio. O stent Multi-Link Pixel ${ }^{\circledR}$ variou seus volumes em 1,23 $\pm 12,59 \mathrm{~mm}^{3}$ entre os períodos pós-procedimento e seguimento, enquanto o stent CardioMind $^{\circledR}$ apresentou variação de 9,18 $\pm 9,77 \mathrm{~mm}^{3}(\mathrm{p}=0,04)$.
A ICP em vasos de fino calibre $(<2,75-2,8 \mathrm{~mm}$ ou $<$ 3,0 mm, dependendo da definição empregada) $)^{15,16}$ tem sido historicamente relacionada a taxas elevadas de reestenose (> 35\%) e revascularização do vasoalvo $(>20 \%)^{17}$.

Estudos randomizados e análises de subgrupos têm demonstrado que a eficácia superior dos stents farmacológicos na redução da proliferação neo-intimal e taxas de reestenose, em comparação aos stents não-farmacológicos, se estende para o subgrupo das intervenções em vasos de fino calibre ${ }^{16,18-24}$. No entanto, o calibre do vaso ainda tem se mostrado forte preditor de reestenose mesmo com o uso dos stents farmacológicos. Em estudo de Kastrati et al. ${ }^{5}$, análise multivariada demonstrou que redução de $0,5 \mathrm{~mm}$ no tamanho do vaso-alvo foi associada a aumento de $70 \%$ nas taxas de reestenose angiográfica e 65\% na revascularização da lesão-alvo após ICP com stent farmacológico. 
TABELA 4

Resultados da análise volumétrica pelo ultra-som intracoronário das bordas proximais

\begin{tabular}{|c|c|c|c|}
\hline & $\begin{array}{l}\text { CardioMind }^{\circledR} \\
\quad n=12\end{array}$ & $\begin{array}{c}\text { Multi-Link Pixel }{ }^{\circledR} \\
\quad n=18\end{array}$ & $\mathbf{p}^{*}$ \\
\hline \multicolumn{4}{|l|}{ Extensão analisada, mm } \\
\hline Pós-procedimento & $4,64 \pm 0,75$ & $4,82 \pm 0,55$ & 0,45 \\
\hline Seguimento & $4,49 \pm 0,96$ & $4,86 \pm 0,48$ & 0,17 \\
\hline $\mathrm{p}^{* *}$ & 0,67 & 0,81 & \\
\hline \multicolumn{4}{|l|}{ Volume do vaso $\left(\mathrm{mm}^{3}\right)$} \\
\hline Pós-procedimento & $42,12 \pm 12,51$ & $53,72 \pm 19,68$ & 0,06 \\
\hline Seguimento & $39,53 \pm 14,72$ & $50,72 \pm 19,47$ & 0,10 \\
\hline $\mathrm{p}^{* *}$ & 0,64 & 0,64 & \\
\hline \multicolumn{4}{|l|}{ Volume da placa $\left(\mathrm{mm}^{3}\right)$} \\
\hline Pós-procedimento & $20,86 \pm 7,20$ & $26,56 \pm 10,81$ & 0,12 \\
\hline Seguimento & $19,84 \pm 8,73$ & $25,60 \pm 10,55$ & 0,13 \\
\hline $\mathrm{p}^{* *}$ & 0,75 & 0,78 & \\
\hline \multicolumn{4}{|c|}{ Volume do lúmen ( $\left.\mathrm{mm}^{3}\right)$} \\
\hline Pós-procedimento & $21,26 \pm 7,01$ & $25,97 \pm 12,59$ & 0,24 \\
\hline Seguimento & $19,69 \pm 7,21$ & $25,13 \pm 13,55$ & 0,21 \\
\hline $\mathrm{p}^{* *}$ & 0,59 & 0,84 & \\
\hline \multicolumn{4}{|c|}{ Área luminal mínima (mm²) } \\
\hline Pós-procedimento & $3,51 \pm 0,83$ & $4,57 \pm 1,81$ & 0,07 \\
\hline Seguimento & $3,30 \pm 1,06$ & $4,26 \pm 1,56$ & 0,07 \\
\hline $\mathrm{p}^{* *}$ & 0,59 & 0,58 & \\
\hline \multicolumn{4}{|l|}{ Carga de placa (\%) } \\
\hline Pós-procedimento & $49,02 \pm 12,07$ & $50,61 \pm 12,54$ & 0,73 \\
\hline Seguimento & $49,01 \pm 9,46$ & $51,37 \pm 12,97$ & 0,59 \\
\hline $\mathrm{p}^{* *}$ & 0,99 & 0,85 & \\
\hline
\end{tabular}

* Teste $t$ de Student não-pareado. ** Teste $t$ de Student pareado.

$\mathrm{n}=$ número de pacientes.

O stent CardioMind ${ }^{\circledR}$, feito de nitinol, liga de níquel e titânio, possui propriedades termoelásticas, que Ihe conferem o fenômeno de "efeito de memória de forma". Após sofrer aparente deformação, recupera sua conformação original depois de cessada a força atuante. Essas propriedades permitem a auto-expansão do stent até um ponto pré-definido ${ }^{25}$.

Com os stents balão-expansíveis, estudos com a utilização de USIC demonstraram que o diâmetro luminal final em vasos com diâmetro menor que 2,75 mm prevê a necessidade de revascularização ${ }^{20}$. Nesse sentido, as altas pressões utilizadas para adequada expansão dessas próteses promovem injúria imediata às camadas profundas da parede vascular ${ }^{26}$, a qual está fortemente correlacionada com a $\mathrm{HNI}$ e o diâmetro de estenose no seguimento mais tardio ${ }^{25}$.

O padrão de injúria à parede do vaso, bem como o momento em que ela ocorre após o implante de stents auto-expansíveis de nitinol, são diferentes daqueles provocados pelos stents balão-expansíveis ${ }^{25,26}$.
Ao passo que os stents balão-expansíveis alcançam seu diâmetro final no momento do implante, os stents auto-expansíveis apresentam típica expansão continuada de seus diâmetros ${ }^{25}$. Em teoria, a capacidade de os stents auto-expansíveis expandirem seus volumes com o passar do tempo pode permitir o implante com pressões mais baixas. Um menor trauma inicial à parede vascular poderia gerar proliferação neo-intimal menos acentuada $^{25,26}$. Entretanto, estudos em animais indicam que a expansão gradual do stent pode prolongar a proliferação neo-intimal por períodos variáveis ${ }^{25,26}$, até mesmo além de três meses, quando comparada a pico mais precoce, entre um e três meses, observado com os stents balão-expansíveis ${ }^{27,28}$.

O presente estudo representa um subestudo do registro SISC $^{9}$, em que procuramos, através da análise seriada com USIC, não só investigar a eficácia do stent CardioMind ${ }^{\circledR}$ em comparação ao stent Multi-Link Pixel ${ }^{\circledR}$, mas também fornecer informações sobre as propriedades mecânicas do funcionamento dessas duas endopróteses e a subseqüente resposta vascular no seg- 
TABELA 5

Resultados da análise volumétrica pelo ultra-som intracoronário das bordas distais

\begin{tabular}{|c|c|c|c|}
\hline & $\begin{array}{l}\text { CardioMind }{ }^{\circledR} \\
\quad \mathbf{n}=10\end{array}$ & $\begin{array}{c}\text { Multi-Link Pixel }{ }^{\circledR} \\
n=20\end{array}$ & $\mathbf{p}^{*}$ \\
\hline \multicolumn{4}{|c|}{ Extensão analisada, mm } \\
\hline Pós-procedimento & $5,0 \pm 0$ & $4,92 \pm 0,36$ & $>0,99$ \\
\hline Seguimento & $4,72 \pm 0,89$ & $4,91 \pm 0,40$ & 0,42 \\
\hline$p^{* *}$ & 0,99 & 0,93 & \\
\hline \multicolumn{4}{|l|}{ Volume do vaso $\left(\mathrm{mm}^{3}\right)$} \\
\hline Pós-procedimento & $31,63 \pm 11,0$ & $39,90 \pm 11,47$ & 0,06 \\
\hline Seguimento & $31,63 \pm 12,64$ & $35,02 \pm 12,87$ & 0,49 \\
\hline$p^{* *}$ & 0,99 & 0,21 & \\
\hline \multicolumn{4}{|l|}{ Volume da placa $\left(\mathrm{mm}^{3}\right)$} \\
\hline Pós-procedimento & $13,92 \pm 6,77$ & $17,20 \pm 8,0$ & 0,27 \\
\hline Seguimento & $14,46 \pm 8,22$ & $15,16 \pm 9,60$ & 0,84 \\
\hline $\mathrm{p}^{* *}$ & 0,87 & 0,46 & \\
\hline \multicolumn{4}{|c|}{ Volume do lúmen (mm³) } \\
\hline Pós-procedimento & $19,00 \pm 6,44$ & $22,67 \pm 7,08$ & 0,17 \\
\hline Seguimento & $17,17 \pm 6,73$ & $18,90 \pm 6,41$ & 0,49 \\
\hline $\mathrm{p}^{* *}$ & 0,54 & 0,08 & \\
\hline \multicolumn{4}{|c|}{ Área luminal mínima (mm²) } \\
\hline Pós-procedimento & $3,11 \pm 1,01$ & $3,83 \pm 1,31$ & 0,13 \\
\hline Seguimento & $2,49 \pm 0,79$ & $3,50 \pm 1,07$ & 0,01 \\
\hline $\mathrm{p}^{* *}$ & 0,14 & 0,38 & \\
\hline \multicolumn{4}{|l|}{ Carga de placa (\%) } \\
\hline Pós-procedimento & $43,66 \pm 12,56$ & $42,47 \pm 12,97$ & 0,81 \\
\hline Seguimento & $44,86 \pm 11,75$ & $41,20 \pm 13,71$ & 0,47 \\
\hline $\mathrm{p}^{* *}$ & 0,82 & 0,76 & \\
\hline
\end{tabular}

* Teste $t$ de Student não-pareado. ** Teste $t$ de Student pareado.

$\mathrm{n}=$ número de pacientes.

mento inteiro da lesão-alvo durante o período médio de acompanhamento.

Algumas considerações com respeito aos aspectos técnicos empregados neste estudo merecem ser feitas. Embora a presente análise não seja randomizada, os pacientes foram selecionados com base nos mesmos critérios de inclusão, e as características clínicas basais foram semelhantes entre os dois grupos. Os pacientes tratados com o stent CardioMind ${ }^{\circledR}$, porém, tinham vasos de diâmetro significativamente menor, assim como a extensão da lesão-alvo. Entretanto, a relação entre o diâmetro dos stents utilizados e o diâmetro de referência do vaso, bem como a extensão do segmento coberto por stent, foram semelhantes nos dois grupos. Nesse sentido, parece que essas discrepâncias iniciais nas características angiográficas foram atenuadas, não interferindo nos resultados finais.

Outro aspecto técnico que merece destaque diz respeito à proporção significativamente maior de realização da pós-dilatação dos stents no grupo Cardio-
Mind $^{\circledR}$. Em nossa instituição, em geral, procuramos realizar adequada pré-dilatação, liberando os stents a baixa pressão (principalmente no tratamento de vasos de fino calibre) e, quando necessário, realizar a pósdilatação, com o uso de balões não-complacentes mais curtos que o stent implantado, com o uso de altas pressões, visando à obtenção de relação 1,1 a 1,2:1,0. Nesse sentido, após o "preparo" da lesão-alvo com a pré-dilatação, a realização de pós-dilatação dos stents balão-expansíveis para otimização de seu implante foi necessária na minoria dos casos. Em contrapartida, o conceito de implante dos stents auto-expansíveis é diferente. O stent liberado "adapta-se" ao segmentoalvo e, ao passo que expande seus diâmetros, exerce força radial contra a parede do vaso, atingindo seu diâmetro luminal final num momento mais tardio. Em função desse fato, apesar da boa pré-dilatação da lesão-alvo, foi necessária a pós-dilatação, em todos os casos, para melhor acomodação dos stents ao segmento-alvo, produzindo lesão residual menor que $20 \%$. Ademais, apesar das diferenças nas técnicas de im- 

com Ultra-Som Intracoronário Tridimensional. Rev Bras Cardiol Invas. 2008;16(3):341-352.

plante, as pressões finais de insuflação de ambas as próteses foram semelhantes nos dois grupos.

Ainda, o efeito de "memória de forma", inerente aos stents auto-expansíveis de nitinol, pode explicar a ocorrência de 6 (46\%) casos de aposição incompleta das hastes do stent CardioMind ${ }^{\circledR}$ imediatamente após seu implante, mesmo com a utilização de pós-dilatação sistemática. Entretanto, sua expansão continuada promoveu resolução da má aposição em 50\% dos casos e não houve aparecimento de nenhum caso de

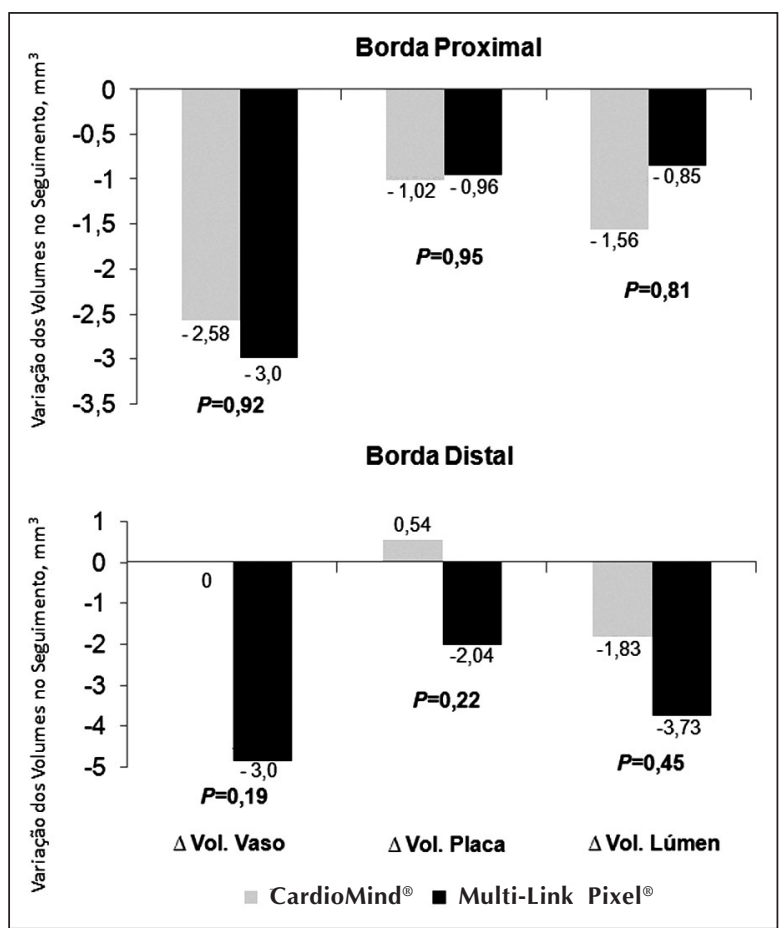

Figura 4 - Representação gráfica das variações volumétricas nos segmentos de bordas proximal e distal dos stents CardioMind ${ }^{\circledast}$ e Multi-Link Pixel ${ }^{\circledast}$ na avaliação tardia com o ultra-som intracoronário. $\Delta$ Vol. = variação do volume. má aposição tardia, fenômeno descrito como preditor de trombose tardia de stents farmacológicos ${ }^{12,13}$.

No que diz respeito à resposta vascular nos segmentos de bordas dos stents, não se notou diferença nas variações volumétricas quando comparadas as bordas proximal e distal dos dois stents. Embora em estudos prévios tenha sido evidenciada menor agressão às bordas com o uso de stent auto-expansível, gerando com isso menos remodelamento naquele segmento ${ }^{26}$, os resultados aqui obtidos estão em consonância com a técnica de implante de stent balão-expansível adotada pela nossa instituição. A política de se utilizar a pré-dilatação com liberação dos stents a baixa pressão e, quando necessário, realizar a pós-dilatação com balões mais curtos, no interior do stent, pode ter evitado qualquer trauma nos bordos das endopróteses.

A utilização de stents auto-expansíveis como plataforma de carreamento de fármacos antiproliferativos já vem sendo testada ${ }^{25,29}$. Em futuro próximo, estará disponível a versão farmacológica do stent CardioMind ${ }^{\circledR}$, impregnado com o fármaco sirolimus em uma matriz polimérica biodegradável.

\section{Limitações}

Este estudo possui algumas limitações que merecem ser mencionadas: (1) o pequeno número da amostra; (2) os grupos de tratamento foram consecutivos, porém não contemporâneos e não randomizados; e (3) pela necessidade inerente a este estudo de comparar USICs seriados (pós-procedimento e no seguimento), houve diminuição do número inicial de pacientes, ao se excluir aqueles que não tiveram USIC realizado nos dois períodos de avaliação, bem como seleção de casos com áreas luminais maiores no seguimento, uma vez que pacientes com reestenoses que impedissem a passagem do cateter de ultra-som também foram excluídos.

\section{CONCLUSÕES}

Os stents CardioMind ${ }^{\circledR}$ produziram volumes de $\mathrm{HNI}$ semelhantes aos do stent Multi-Link Pixel ${ }^{\circledR}$. Entretanto,

TABELA 6

Comparação da variação seriada dos volumes entre as bordas proximal e distal dos stents CardioMind ${ }^{\circledR}$ e Multi-Link Pixe ${ }^{\circledR}$

\begin{tabular}{|c|c|c|c|c|c|c|}
\hline & \multicolumn{3}{|c|}{ CardioMind $^{\circledR}$} & \multicolumn{3}{|c|}{ Multi-Link Pixel ${ }^{\circledR}$} \\
\hline & $\begin{array}{c}\text { Borda } \\
\text { proximal }\end{array}$ & $\begin{array}{c}\text { Borda } \\
\text { distal }\end{array}$ & $\mathbf{p}$ & $\begin{array}{c}\text { Borda } \\
\text { proximal }\end{array}$ & $\begin{array}{c}\text { Borda } \\
\text { distal }\end{array}$ & $p$ \\
\hline$\Delta$ Vol. vaso & $-2,58 \pm 3,96$ & $0 \pm 9,12$ & 0,38 & $-3,0 \pm 14,99$ & $-4,87 \pm 9,70$ & 0,64 \\
\hline$\Delta$ Vol. placa & $-1,02 \pm 3,27$ & $0,54 \pm 1,81$ & 0,19 & $-0,96 \pm 8,20$ & $-2,04 \pm 6,37$ & 0,65 \\
\hline$\Delta$ Vol. lúmen & $-1,56 \pm 3,34$ & $-1,83 \pm 6,11$ & 0,89 & $-0,85 \pm 9,94$ & $-3,76 \pm 6,89$ & 0,29 \\
\hline$\Delta \mathrm{ALM}$ & $-0,21 \pm 0,77$ & $-0,63 \pm 1,30$ & 0,35 & $-0,32 \pm 1,38$ & $0,05 \pm 1,86$ & 0,49 \\
\hline$\Delta$ Carga placa & $-0,01 \pm 7,12$ & $1,21 \pm 10,80$ & 0,75 & $0,76 \pm 11,76$ & $-1,27 \pm 8,03$ & 0,53 \\
\hline
\end{tabular}

ALM = área luminal mínima; Vol. = volume. 

com Ultra-Som Intracoronário Tridimensional. Rev Bras Cardiol Invas. 2008;16(3):341-352.

apresentaram aumento em suas dimensões significativamente maior ao observado com o Multi-Link Pixel ${ }^{\circledR}$, promovendo melhor acomodação do volume de hiperplasia, com menor porcentual de obstrução volumétrica. Nenhum dos dois tipos de stent causou injúria aos segmentos das bordas.

\section{REFERÊNCIAS BIBLIOGRÁFICAS}

1. Foley DP, Melkert R, Serruys PW. Influence of coronary vessel size on renarrowing process and late angiographic outcome after successful balloon angioplasty. Circulation. 1994;90(3):1239-51.

2. Elezi S, Kastrati A, Neumann FJ, Hadamitzky M, Dirschinger J, Schömig A. Vessel size and long-term outcome after coronary stent placement. Circulation. 1998;98(18):1875-80.

3. Hsieh IC, Chien CC, Chang HJ, Chern MS, Hung KC, Lin $\mathrm{FC}$, et al. Acute and long-term outcomes of stenting in coronary vessel $>3.0 \mathrm{~mm}, 3.0-2.5 \mathrm{~mm}$, and $<2.5 \mathrm{~mm}$. Catheter Cardiovasc Interv. 2001;53(3):314-22.

4. Holmes DR Jr, Leon MB, Moses JW, Popma JJ, Cutlip D, Fitzgerald PJ, et al. Analysis of 1-year clinical outcomes in the SIRIUS trial: a randomized trial of a sirolimus-eluting stent versus a standard stent in patients at high risk for coronary restenosis. Circulation. 2004;109(5):634-40.

5. Kastrati A, Dibra A, Mehili J, Mayer S, Pinieck S, Pache J, et al. Predictive factors of restenosis after coronary implantation of sirolimus- or paclitaxel-eluting stents. Circulation. $2006 ; 113(19): 2293-300$.

6. Morice MC. Stenting for small coronary vessels. J Invasive Cardiol. 2003;15(7):377-9.

7. Abizaid A, Costa Jr JR, Whitbourn RJ, Chang JC. The CardioMind ${ }^{\mathrm{TM}}$ coronary stent delivery system: stent delivery on a .014" guidewire platform. Eurolntervention. 2007;3:154-7.

8. Chamié D, Costa Jr JR, Abizaid A, Gottschald MF, Feres F, Mattos LA, et al. Exeqüibilidade, eficácia e segurança do sistema de stent CardioMind ${ }^{\mathrm{TM}}$ no tratamento de lesões em vasos de fino calibre. Um estudo seriado com angiografia e ultra-som intracoronário. Rev Bras Cardiol Invas. 2008;16(2):193-9.

9. Chamié D, Costa Jr JR, Abizaid A, Devito F, Feres F, Gottschald $M$, et al. Angiographic and ultrasonographic results of the SISC (Stents In Small Coronaries) registry. J Am Coll Cardiol Interv. 2008;1(2 Suppl B):B9.

10. Mintz GS, Nissen SE, Anderson WD, Bailey SR, Erbel R, Fitzgerald PJ, et al. American College of Cardiology Clinical Expert Consensus Document on Standards for Acquisition, Measurement and Reporting of Intravascular Ultrasound Studies (IVUS). A report of the American College of Cardiology Task Force on Clinical Expert Consensus Documents. J Am Coll Cardiol. 2001;37(5):1478-92.

11. Mehran R, Mintz GS, Hong MK, Tio FO, Bramwell O, Brahimi $A$, et al. Validation of the in vivo intravascular ultrasound measurement on in-stent neointimal hyperplasia volumes. J Am Coll Cardiol. 1998;32(3):794-9.

12. Cook $S$, Wenaweser $P$, Togni $M$, Billinger $M$, Morger $C$, Seiler $\mathrm{C}$, et al. Incomplete stent apposition and very late stent thrombosis after drug-eluting stent implantation. Circulation. 2007;115(18):2426-34.

13. Siqueira DA, Abizaid AA, Costa JR, Feres F, Mattos LA, Staico $R$, et al. Late incomplete apposition after drugeluting stent implantation: incidence and potential for adverse clinical outcomes. Eur Heart J. 2007;28(11):1304-9.

14. Smith SC Jr, Dove JT, Jacobs AK, Kennedy JW, Kereiakes D, Kern MJ, et al. ACC/AHA guidelines for percutaneous coro- nary intervention (revision of the 1993 PTCA guidelines)executive summary: a report of the American College of Cardiology/American Heart Association task force on practice guidelines (Committee to revise the 1993 guidelines for percutaneous transluminal coronary angioplasty) endorsed by the Society for Cardiac Angiography and Interventions. Circulation. 2001;103(24):3019-41.

15. Moreno R, Fernández C, Alfonso F, Hernández R, Pérez-Vizcayno MJ, Escaned J, et al. Coronary stenting versus balloon angioplasty in small vessels: a meta-analysis from 11 randomized studies. J Am Coll Cardiol. 2004;43(11):1964-72.

16. Ardissino D, Cavallini C, Bramucci E, Indolfi C, Marzocchi A, Manari A, et al. Sirolimus-eluting vs uncoated stents for prevention of restenosis in small coronary arteries: a randomized trial. JAMA. 2004;292(22):2727-34.

17. Mehilli J, Dibra A, Kastrati A, Pache J, Dirschinger J, Schömig A. Randomized trial of paclitaxel- and sirolimus-eluting stents in small coronary vessels. Eur Heart J. 2006;27(3):260-6.

18. Nikolsky E, Moses JW, Cambier P, Bachinsky B, O'Shaughnessy C, Mehran R, et al. Results from the $2.25 \mathrm{~mm}$ Sirolimuseluting Bx Velocity Stent Registry in patients with native coronary artery lesions. Am J Cardiol. 2005;96 Suppl 7A:179H.

19. Regar E, Serruys PW, Bode C, Holubarsch C, Guermonprez JL, Wijns W, et al. Angiographic findings of the multicenter Randomized Study with the Sirolimus-Eluting Bx Velocity Balloon-Expandable Stent (RAVEL): sirolimus-eluting stents inhibit restenosis irrespective of the vessel size. Circulation. 2002;106(15):1949-56.

20. lakovou I, Mintz GS, Dangas G, Abizaid A, Mehran R, Lansky AJ, et al. Optimal final lumen area and predictors of target lesion revascularization after stent implantation in small coronary arteries. Am J Cardiol. 2003;92(10):1171-6.

21. Schampaert E, Cohen EA, Schlüter $M$, Reeves $F$, Traboulsi $M$, Title LM, et al. The Canadian study of the sirolimus-eluting stent in the treatment of patients with long de novo lesions in small native coronary arteries (C-SIRIUS). J Am Coll Cardiol. 2004;43(6):1110-5.

22. Schofer J, Schlüter M, Gershlick AH, Wijns W, Garcia E, Schampaert E, et al. Sirolimus-eluting stents for treatment of patients with long atherosclerotic lesions in small coronary arteries: double-blind, randomised controlled trial (E-SIRIUS). Lancet. 2003;362(9390):1093-9.

23. Meier B, Sousa E, Guagliumi G, Van den Branden F, Grenadier $\mathrm{E}$, Windecker $\mathrm{S}$, et al. Sirolimus-eluting coronary stents in small vessels. Am Heart J. 2006;151(5):1019.e1-7.

24. Lemos PA, Arampatzis CA, Saia F, Hoye A, Degertekin M, Tanabe $\mathrm{K}$, et al. Treatment of very small vessels with 2.25 $\mathrm{mm}$ diameter sirolimus-eluting stents (from the RESEARCH registry). Am J Cardiol. 2004;93(5):633-6.

25. Cilingiroglu M, Elliott J, Patel D, Tio F, Matthews H, McCasland $M$, et al. Long-term effects of novel biolimus-eluting DEVAX AXXESS plus nitinol self-expanding stent in a porcine coronary model. Catheter Cardiovasc Interv. 2006;68(2):271-9.

26. Kobayashi Y, Honda Y, Christie GL, Teirstein PS, Bailey $S R$, Brown CL $3^{\text {rd }}$, et al. Long-term vessel response to a selfexpanding coronary stent: a serial volumetric intravascular ultrasound analysis from the ASSURE Trial. J Am Coll Cardiol. 2001;37(5):1329-34.

27. Hong MK, Beyar R, Kornowski R, Tio FO, Bramwell O, Leon MB. Acute and chronic effects of self-expanding nitinol stents in porcine coronary arteries. Coron Artery Dis. 1997;8(1):45-8.

28. Beyar R, Henry M, Shofti R, Grenedier E, Globerman O, Beyar M. Self-expandable Nitinol stent for cardiovascular applications: canine and human experience. Cathet Cardiovasc Diagn. 1994;32(2):162-70.

29. Verheye S, Trauthen B. Axxess ${ }^{\mathrm{TM}}$ biolimus A9 eluting bifurcation stent system. Eurolntervention. 2007;2:506-8. 